\title{
Preventing Implant Bacterial Infections with Interconnected 3D Porous Structures (I3D)- A Proof-of-Concept Study
}

\author{
Steven Guo ${ }^{1,2}$, Alexandria Ramos ${ }^{1}$, Zhongqiang $\mathrm{Li}^{3}$, Hong $\mathrm{Yao}^{4}$, Jian $\mathrm{Xu}^{3}$, Shaomian Yao ${ }^{1}$ \\ ${ }^{1}$ Comparative Biomedical Sciences, School of Veterinary Medicine, Louisiana State University, Baton Rouge, LA, USA \\ ${ }^{2}$ Department of Biomedical Engineering, Johns Hopkins University, Baltimore, MD, USA \\ ${ }^{3}$ Division of Electrical and Computer Engineering, College of Engineering, Louisiana State University, Baton Rouge, LA, USA \\ ${ }^{4}$ Department of Mechanical and Industrial Engineering, Louisiana State University, Baton Rouge, LA, USA \\ Email:shaomia@lsu.edu
}

How to cite this paper: Guo, S., Ramos, A., Li, Z.Q., Yao, H., Xu, J. and Yao, S.M. (2021) Preventing Implant Bacterial Infections with Interconnected 3D Porous Structures (I3D)-A Proof-of-Concept Study. Journal of Biosciences and Medicines, 9, 16-29. https://doi.org/10.4236/jbm.2021.91002

Received: December 5, 2020

Accepted: January 10, 2021

Published: January 13, 2021

Copyright $\odot 2021$ by author(s) and Scientific Research Publishing Inc. This work is licensed under the Creative Commons Attribution International License (CC BY 4.0).

http://creativecommons.org/licenses/by/4.0/

\begin{abstract}
Oral antibiotics are often prescribed to prevent infection after implant surgery; however, only a small fraction of the antibiotics can reach the implants. Thus, there are concerns about overusing antibiotics. We designed and fabricated porous implants with interconnecting 3D structures (I3D) and hypothesized that such I3D structures could serve as a depository for antimicrobial agents to prevent infection locally. The implants were either treated with antibiotics or coated with silver nanoparticles (AgNPs) by electrodeposition to test this hypothesis. The antimicrobial assay was conducted, and bacterial growth zones of inhibition (ZOIs) were monitored. Overall, I3D implants resulted in larger ZOIs than did the solid implants, and the center I3D (cI3D)-implant produced the largest ZOI. In the antibiotic treatment testing, the diameters of ZOIs of the solid implant vs. I3D implant were about $14 \mathrm{~mm}$ vs. 15 to $18 \mathrm{~mm}$ on day 2 ; however, the diameter quickly reduced to $9 \mathrm{~mm}$ on day 3 and $5 \mathrm{~mm}$ on days 6 and 8 for the solid implant, while no obvious change of the zone was seen for I3D implants. For the AgNPs coated implants, the ZOIs for the I3D implants were generally greater than the solid implant over four weeks of incubation. A significantly larger ZOI $(\sim 1-2 \mathrm{~mm}$ larger on average) was seen for AgNPs coated I3D implants at $0.1 \mathrm{~V}-0.01 \mathrm{M}$, $0.3 \mathrm{~V}-0.01 \mathrm{M}$, and $1.5 \mathrm{~V}-0.01 \mathrm{M}$ treatments compared to AgNPs coated solid implants. Given that we have previously shown that I3D implants can reserve chemoattractants to recruit stem cells to enhance osseointegration, we conclude that implants with the I3D structures could be beneficial not only for osseointegration but also in preventing infection.
\end{abstract}




\section{Keywords}

Dental Implant, Antimicrobial Agents, Peri-Implantitis, Silver Nanoparticles, 3D-Printing

\section{Introduction}

Dental implants are currently the most effective therapy for missing teeth. After surgical installation, the implants must be connected to the surrounding bone through osseointegration. One of the leading causes of implant failure is the development of peri-implantitis, a resultant of bacterial infection. With the mouth being considered an inherently dirty field with high incidences of bacteremia [1], preventing the onset of infection in any surgical wound is especially critical for the success of dental implants. Prophylactic oral antibiotics are often prescribed pre- or post-operatively to achieve an antibiotic concentration in the blood to prevent bacterial proliferation and dissemination [2]; however, this raises a concern of overusing/overdosing antibiotics which would produce a greater difficulty in treating legitimate infection due to increased antibiotic resistance [3] [4].

Two implant-related diseases: peri-mucositis and peri-implantitis (known asperi-implant diseases), are common in current dental implants [5]. Peri-mucositis, found in nearly $48 \%$ of all implants [6], occurs in the soft tissues encircling a dental implant and can lead to peri-implantitis [7], a destructive disease that affects the supportive bone around the implants. In the process of disease development, the association of glycoproteins from saliva with microbiological colonization was found to be a main cause for the formation of a biofilm on an implant's surface. This process of a biofilm formation occurs shortly after implants are placed [8] [9] [10]. Studies showed that Gramnegative anaerobic bacteria are the culprits for the development of peri-implant diseases [11], and that the formation of the biofilm plays a vital role in the development of infections. Thus, preventing attachment of bacteria on the implants after surgery is especially critical to avoid biofilm formation.

Therefore, preventing the attachment of the bacteria to the implants' surface for the formation of microbiological colonization is critical to prevent the development of peri-implant diseases. Titanium implants are commonly used in the medical and dental fields with a high success rate. Previously, we reported using additive manufacturing (or $3 \mathrm{D}$ printing) to conveniently fabricate titanium implants with interconnecting 3D porous structures (I3D). We have shown that such porous structure of the I3D implants can serve as a depository for chemoattractant to recruit stem cells to enhance osseointegration [12]. We reasoned that such porous structures of the implants could also serve as a reservoir for antimicrobial agents. And the antimicrobial agents reserved in the porous structures can be slowly released to prevent bacterial growth around and at- 
tachment to the implants upon surgical installation. With this in mind, we revised the $\mathrm{I} 3 \mathrm{D}$ structures by varying different porous interconnecting patterns (Figure $1(\mathrm{~A})$ ). The objective of this study was to determine the optimal I3D structures for storing antimicrobial agents to achieve antimicrobial capability in vitro.

Penicillin and streptomycin had been reported for coating on titanium with polypyrrole for slow releasing for orthopedic applications [13]. Penicillin is a highly effective and inexpensive antimicrobial of choice for the initial treatment of odontogenic infections [14]. Silver has long been known to have antibacterial activity with low toxicity to human cells and has been widely used for the treatment of burns. Recently, silver nanoparticles (AgNPs) have received attention for clinical and therapeutic applications because of their antimicrobial activity against both Gram-positive and Gram-negative bacteria [15]. It was reported that AgNPs possess a broad spectrum of antibacterial and antifungal activities [16] [17] [18] [19] [20] with less biological activities/toxicity than silver ions in human cells [21] [22]. In this project, we tested the hypothesis that the patterns of I3D structures play a critical role in preserving and releasing antimicrobial agents against bacteria. Specifically, titanium implants were fabricated using LPBF-based 3D printing. We first explored to treat implants with penicillin and streptomycin. An optimal I3D implant was identified for coating with silver nanoparticles (AgNPs) using an electrodeposition method based on the antibiotics

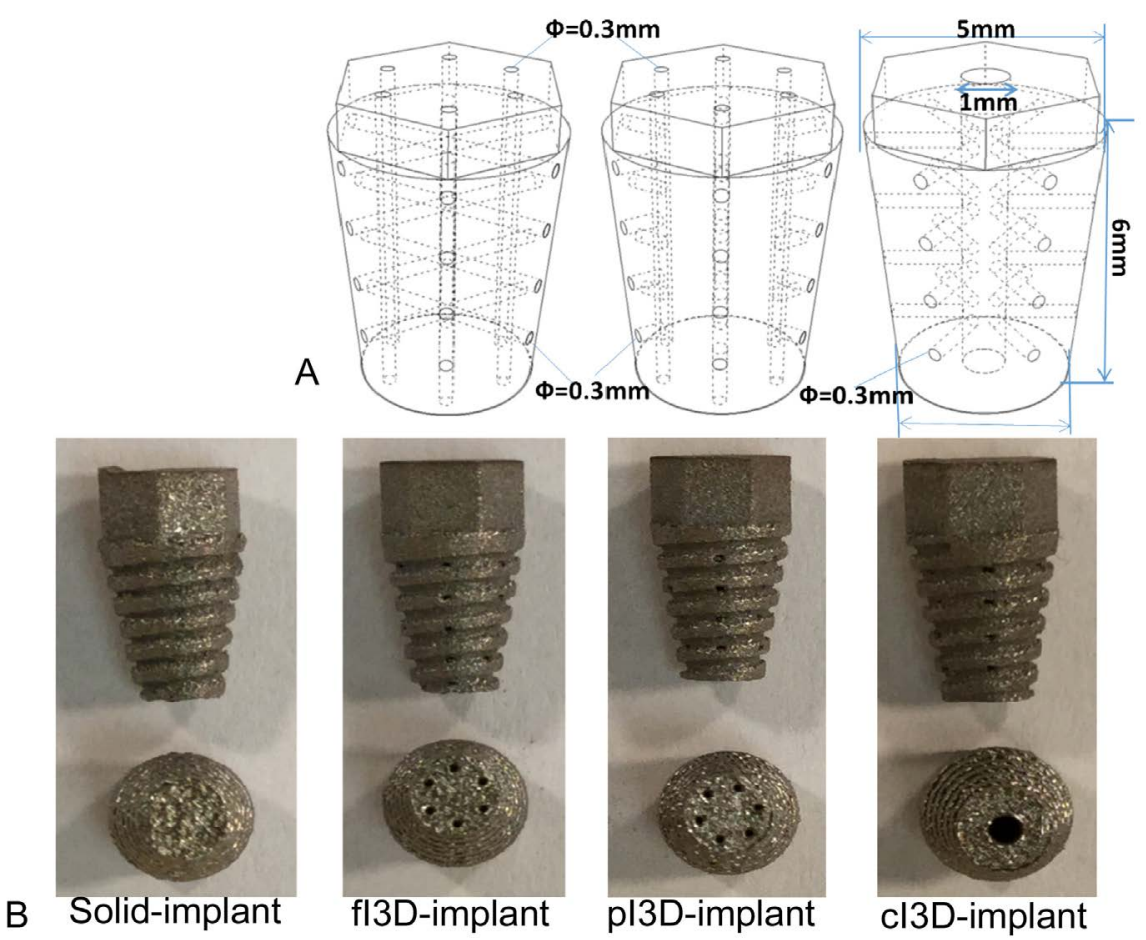

Figure 1. Porous implant design and fabrication of the implants. (A) The implant designs showing various interconnecting $3 \mathrm{D}$ porous structures (I3D) of the implants, namely full I3D (fI3D) implant, partial I3D (pI3D) implant, and center I3D (cI3D) implant. (B) Titanium implants fabricated with $3 \mathrm{D}$ laser printing showing pores at side and bottom views. 
experiment. In vitro antimicrobial assay was carried out with LB-agar plates inoculated with $E$. coli bacteria to evaluate the bacterial growth inhibition of the implants coated with either penicillin/streptomycin or AgNPs. The results of this study will not only shed light on future implant designs, fabrication, and applications, but also on post-surgery care to prevent infection and peri-implantitis.

\section{Materials and Methods}

\subsection{Implant Designs and Fabrication}

We designed implants with 3D porous interconnecting structures, namely interconnecting 3D threaded (I3D) structures. In particular, the porous structures are interconnected in different patterns and we named the implants full I3D (fI3D) implant, partial I3D (pI3D) implant, and center I3D (cI3D) implant as shown in Figure 1(A). The diameter of the interconnecting pores of the I3D implant design was $0.3 \mathrm{~mm}$ because the previous study suggested that the pore size of $0.2-0.3 \mathrm{~mm}$ was optimal for osseointegration [12]. A Concept Laser Mlab-cusing-R LPBF system was used to prepare the implants using Titanium TiAl6V4 powders (Concept-Laser) with "Speed-cusing" processing parameters. The 3D-printed implants have the designed features and threaded structures as shown in Figure 1(B).

\subsection{Coating of Implants with Antimicrobial Agents}

To coat antibiotics, implants were sterilized by autoclaving $\left(121^{\circ} \mathrm{C}\right.$ for $30 \mathrm{mi}-$ nutes) and cooled down to room temperature. Implants were then dipped in an antibiotic solution consisting of penicillin $500 \mathrm{units} / \mathrm{ml}+$ streptomycin $500 \mu \mathrm{g} / \mathrm{ml}$ for about 10 seconds. Next, the implants were air-dried (Figure 2(A)) in a Biosafety Cabinet.

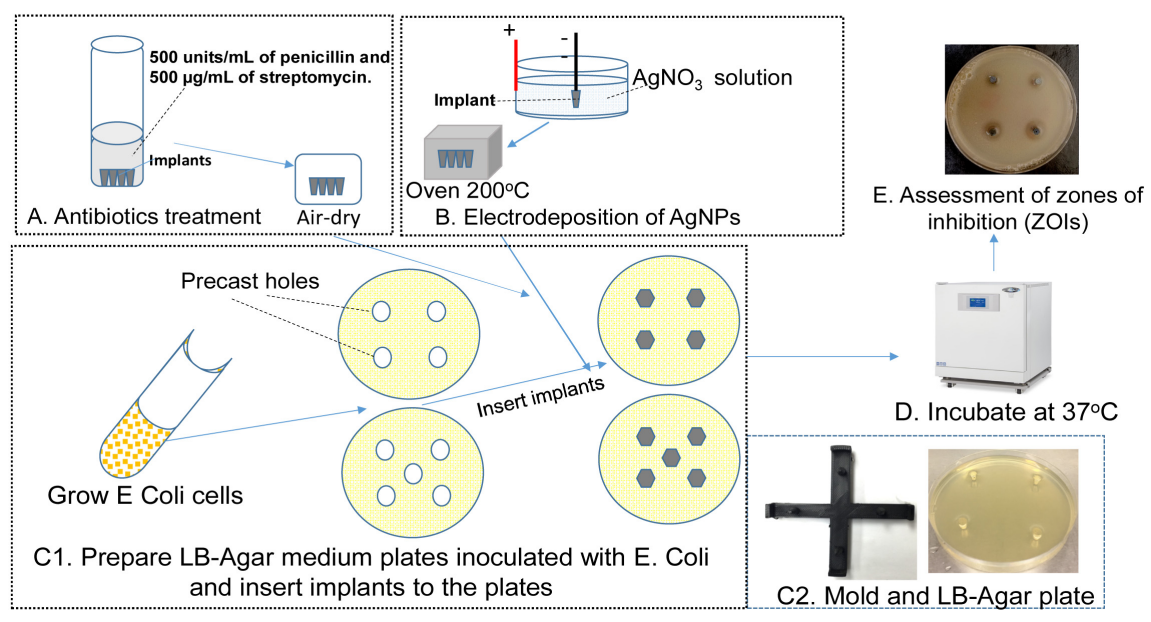

Figure 2. Schematic illustration of the experimental procedures: (A) Antibiotics treatment; (B) Electrodeposition of AgNPs; (C) Preparation of LB-Agar medium plates inoculated with E Coli for insertion of implants. Note that the mold for precast holes and example of the LB-Agar plate with precast holes are shown in C2; (D) Incubation of the plates; (E) assessment of zones of inhibition (ZOIs). 
Electrodeposition was performed to coat silver nanoparticles (AgNPs) on the implants using a method modified from a publication [23]. A homemade electrodeposition system consisting of an electrolyte tank, electrodes (anode: alligator clamps attached with platinum wire; cathode: a platinum wire encircled the tank), and an adjustable DC power supply was used to perform all electrodeposition treatments. The tank was filled with silver nitrate $\left(\mathrm{AgNO}_{3}\right)$ solution, and implant was attached to the anode with the clamp at the center of the tank. The electrodes were connected to a DC power supply (Figure 2(B)). We tested different voltages and concentrations of $\mathrm{AgNO}_{3}$ to optimize the electrodeposition parameters for the duration of one minute to coat implants with AgNPs. The implants were then baked at $200^{\circ} \mathrm{C}$ in an oven for one hour.

\subsection{Antimicrobial Test of the Implants}

A laboratory $E$. coli strain was grown in $5 \mathrm{ml} \mathrm{LB}$ medium in a $15 \mathrm{ml}$-centrifuge tube in a shaker overnight at $37^{\circ} \mathrm{C}, 250 \mathrm{RPM}$ to an OD600 of approximate 1.5. LB medium containing $1 \%$ agar was autoclaved. When the medium was cool enough, $500 \mu \mathrm{l}$ of the $E$. coli suspension was added to $250 \mathrm{ml} \mathrm{LB}$ medium and mixed well. The medium was poured into $100 \mathrm{~mm}$ Petri dishes at about $25 \mathrm{ml}$ per dish. Plastic molds (generated by 3D printing) identical to the shape of the titanium implants were placed to each of the dishes before the gelling of the agar medium. When the medium was cooled down to the room temperature, the molds were carefully removed from the plates leaving the holes identical to the implants on the agar plates (Figure 2(C)). Next, the implants were inserted into the holes of the agar plates. The plates were wrapped with parafilm and incubated at $37^{\circ} \mathrm{C}$ for the formation of zones of bacterial growth inhibition (ZOIs) (Figure 2(D) and Figure 2(E)).

\subsection{Measurement of the Zones of Inhibition (ZOIs)}

The LB agar plates with implants were monitored for the ZOIs. The plates were photographed at the designated time for up to 4 weeks. All ZOI diameters were measured in relation to the known diameter of each petri-dish $(100 \mathrm{~mm})$ using the NIH ImageJ program. It should be noticed that the diameters of the ZOIs include the diameter of the implants, which is $5 \mathrm{~mm}$. Thus, when the ZOI is $5 \mathrm{~mm}$, there is no growth inhibition formed (Figure 3 ).

\subsection{Statistical Analysis}

Statistical Analysis Software (SAS) program mixed model was used to calculate analysis of variance (ANOVA), and LSD was performed for statistical comparison of more than two means. For comparisons of two means, Student t-test was conducted. Statistical significance was achieved when $\mathrm{P} \leq 0.05$.

\section{Results}

The titanium implants fabricated with LPBF-based 3D printing have designed 


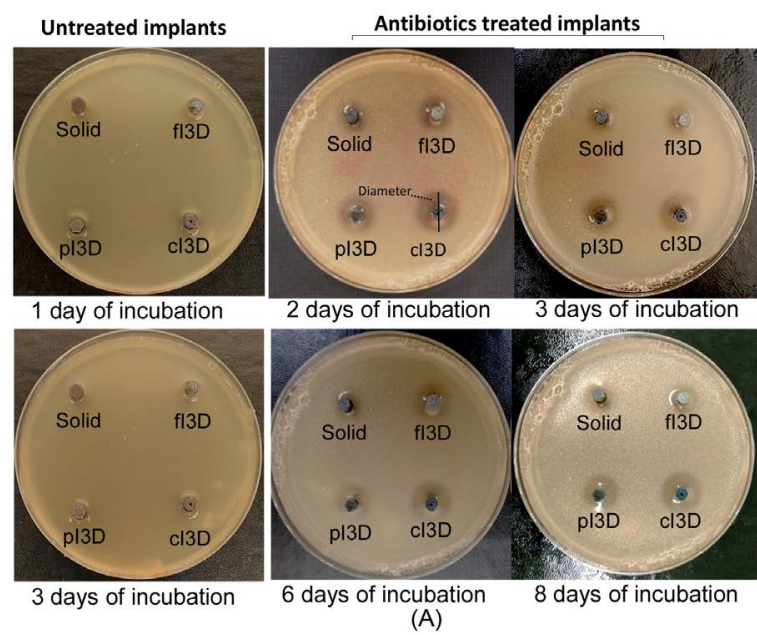

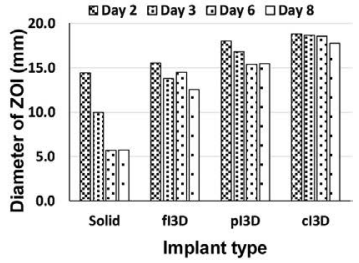

(B)

Figure 3. ZOIs from the antimicrobial assay of the implants coated antibiotics. (A) Note that no ZOIs were observed for the control implants without antibiotic treatment (Left panel). ZOIs were seen for all implants treated with antibiotics. However, I3D implants treated with antibiotics showed larger ZOIs than the solid implant (Right panel). (B) Comparison of solid and I3D implants coasted with Pen/strep on the formation of the ZOIs. Note that the ZOI of the solid implant was quickly reduced, whereas the ZOIs of I3D implants remained relatively constant over time, indicating the sustained releasing of the antibiotics from the I3D implants.

thread and porous features, as shown in Figure 1(B). To test our hypothesis that the porous I3D structures can serve as a depository for antimicrobial reagents, we treated the implants with antibiotics solution (penicillin + streptomycin) and then performed antibacterial assay as described above. Bacterial ZOIs were observed in all implants treated with antibiotics (Figure 3(A) right panel), whereas bacterial growth (i.e., no ZOI) was seen around the control implants without antibiotics treatment after only 1 day of incubation at $37^{\circ} \mathrm{C}$ (Figure 3(A) left panel). We noticed that all I3Dimplantsresulted in larger visible ZOIs than that of the solid implant when coated with antibiotics. Among I3D implants, the cI3D implant had the maximal ZOIs followed by the pI3D implant. Importantly, the ZOIs remained relatively constant for all three types of the I3D implants, whereas the ZOI was greatly reduced for the solid implant over the testing time (Figure 3). Numerically, the I3D implants were seen to increase antimicrobial resistance by $\sim 30 \%$ in comparison to the solid implants by day 2 . And by days 6 and 8 , no obvious ZOI could be seen around the solid implant, i.e., ZOI diameter was about the same as the implant diameter $(5 \mathrm{~mm})$. In contrast, ZOIs were clearly shown in all I3D implants (Figure 3 ) on day 8 of incubation.

Given the superior performance of the cI3D implant in antibiotics treatments and long been known antimicrobial property of silver, we coated cI3D and solid implants with silver nanoparticles (AgNPs) by electrodeposition (Figure 4) and tested antimicrobial activities of the coated implants for four weeks. ZOIs were also observed in all implants coated with AgNPs. We studied the effect of electrodeposition voltages and $\mathrm{AgNO}_{3}$ concentrations for depositing AgNPs on implants. The antimicrobial assay showed that apparent ZOIs could still be seen 


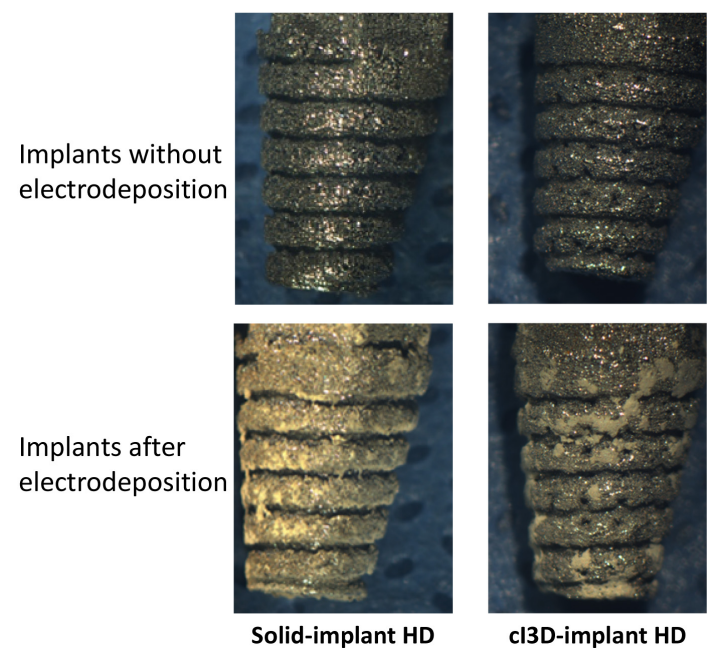

Figure 4. Electrodeposition to coat the implants with AgNPs. Note that the AgNPs coating was shown on the implants subjected to electrodeposition whereas no coating was seen in the controls without electrodeposition.

after four weeks of incubation in bacterial LB-agar plates (Figure 5). The diameters of ZOIs to compare solid vs. porous cI3Dimplants coated with AgNPs by electrodeposition treatments are shown in Figure 6. In general, cI3D implants resulted in larger ZOIs than the solid implants regardless of electrodeposition treatments; however, significant larger ZOIs were seen for the cI3D implants compared to the solid implants when both were treated with $\mathrm{AgNO}_{3} 0.01$ $\mathrm{M}$ at the electrodeposition voltages of $0.1 \mathrm{~V}, 0.3 \mathrm{~V}$ or $1.5 \mathrm{~V}$. Noticeably, the maximal ZOIs were observed for cI3D implants with $0.2 \mathrm{M} \mathrm{AgNO}_{3}$ at $0.3 \mathrm{~V}$ and $0.5 \mathrm{~V}$ electrodeposition (Figure 6).

Comparing the combination effect of $\mathrm{AgNO}_{3}$ concentration and electrodeposition voltage, we found no significant differences on the solid implants (Figure 7). In contrast, for cI3D implants, electrodeposition at $0.3-0.5 \mathrm{~V}$ with $0.2 \mathrm{M}$ $\mathrm{AgNO}_{3}$ showed significantly larger ZOIs than $0.1 \mathrm{~V}-0.01 \mathrm{M} \mathrm{AgNO}_{3}$ (Figure 7), indicating that $0.3-0.5 \mathrm{~V}$ with $0.2 \mathrm{M} \mathrm{AgNO}_{3}$ was superior to $0.1 \mathrm{~V}-0.01 \mathrm{M}$ $\mathrm{AgNO}_{3}$ for electroporation of AgNPs. Although the electrodeposition of AgNPs at $0.2 \mathrm{M} \mathrm{AgNO}_{3}$ yielded maximal ZOIs for both solid and cI3D implants, especially at voltages of 0.3 and $0.5 \mathrm{~V}$, there were interactions between $\mathrm{AgNO}_{3}$ concentration and voltages (Figure 8). A low concentration of $\mathrm{AgNO}_{3}$, such as 0.01 $\mathrm{M}$ required a higher voltage to achieve better electrode position outcomes; however, the interaction effect was not statistically significant.

\section{Discussion}

Infection remains the most common complication for dental implants with approximately half of all patients experiencing some degree of infection. With the sheer magnitude of bacterium inhabiting the mouth, infection could happen at any time whether it is right after implant surgery, or even years after. As current 


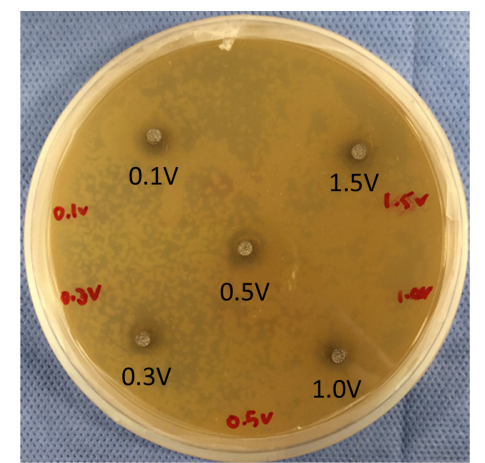

Solid implant

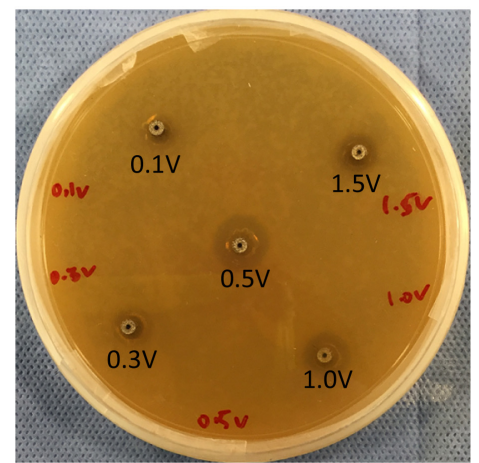

cl3D implant

Figure 5. ZOIs from the antimicrobial assay of the implants coated AgNPs by electrodeposition. Note that the cI3D implants showed generally larger ZOIs than the solid implant. Photos shown were the plates after four weeks of incubation at $37^{\circ} \mathrm{C}$.

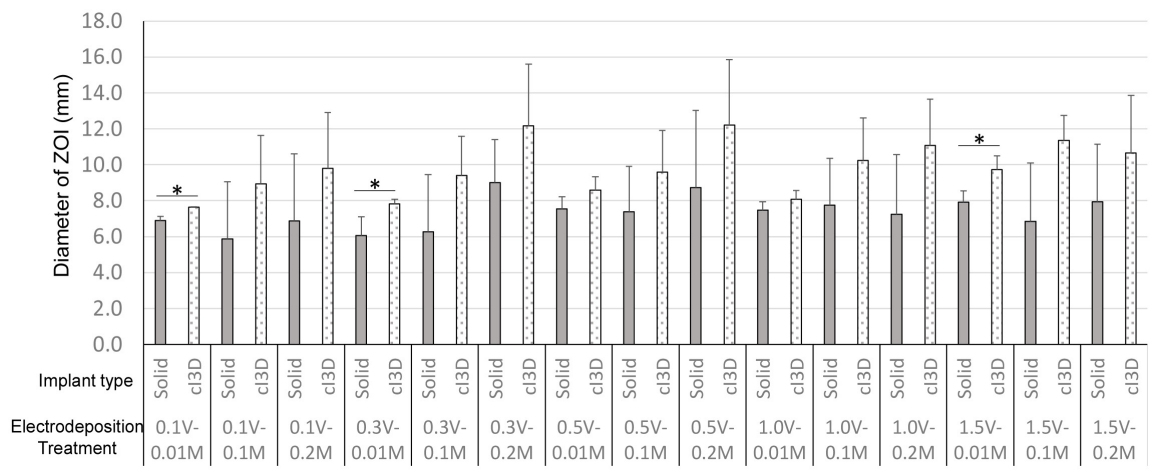

Figure 6. Comparison of solid vs. porous (cI3D)-implants coated with AgNPs by electrodeposition on bacterial growth inhibition. Data shown were the diameter of ZOIs of solid and cI3D implants after four weeks of incubation. Note that, in general, larger ZOIs were formed in cI3D implants than solid implants within each electrodeposition treatment. ${ }^{*}$ indicates that the cI3D implant resulted in a statistically significant larger ZOI than the Solid implant within the treatment group at $\mathrm{P} \leq 0.05$.

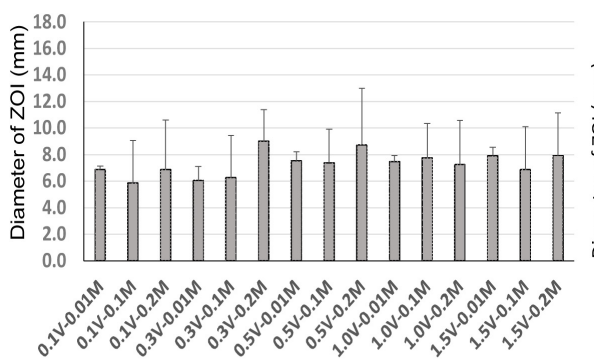

A-solid

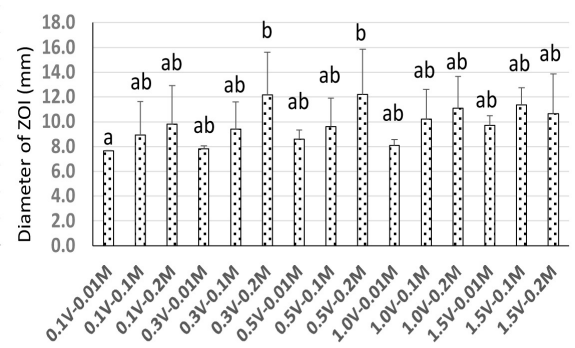

B- $C l 3 D$

Figure 7. Comparison of the combined effects of electrodeposition voltage and $\mathrm{AgNO}_{3}$ concentration for coating solid- and cI3D implants. (A) The diameters of ZOIs of solid implants coated with AgNPs after 4 weeks of incubation; (B) The diameters of ZOIs of cI3D implants coated with AgNPs after 4 weeks of incubation. Note that the voltage and $\mathrm{AgNO}_{3}$ concentration did not affect the ZOI of the solid implant. And 0.3 V, 0.2 M Ag$\mathrm{NO}_{3}$ and $0.5 \mathrm{~V} 0.2 \mathrm{M} \mathrm{AgNO}_{3}$ resulted in significantly larger $\mathrm{ZOI}$ than $0.1 \mathrm{~V} 0.01 \mathrm{M} \mathrm{Ag}$ $\mathrm{NO}_{3}$ for cI3D implant. 

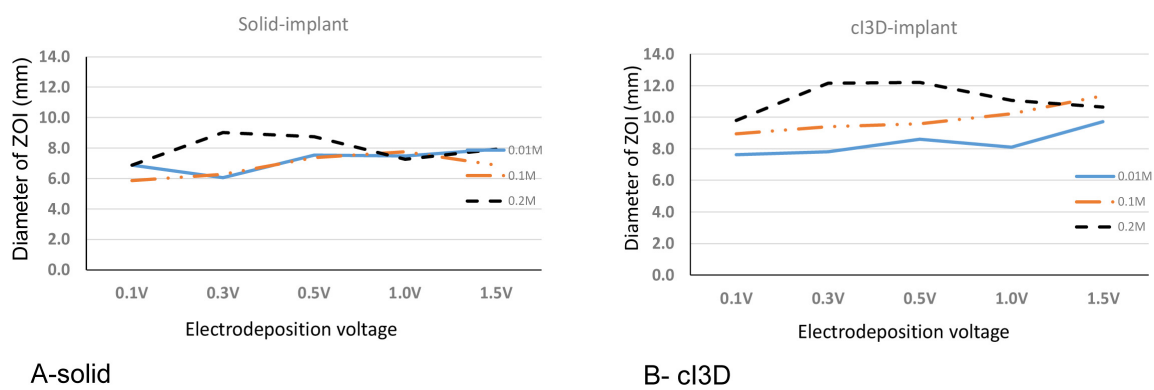

Figure 8. The interaction effects of electrodeposition voltage and $\mathrm{AgNO}_{3}$ concentration for coating solid and cI3D implants. Data shown were the ZOIs of solid implants (A) and cI3D implants (B) coated with AgNPs after four weeks of incubation. For both types of implants, electrodeposition at $0.3 \mathrm{~V}-0.5 \mathrm{~V}$ with $0.2 \mathrm{M} \mathrm{AgNO}_{3}$ solution yielded the largest ZOI.

standards of resolving these issues through oral antibiotics lead only to more concerns with antibiotic overuse, this study gives a first glimpse at a new solution. An interconnecting porous structure (I3D) designed to be used as a local depository that maximizes the effectiveness of antibiotics at the minimum amount used. When these implants are used in dental practices, it is expected that the slow release of these antibiotics stored in the I3D structures can prevent infection far after surgery.

Silver was one of the variables we tested for coating implants to achieve antimicrobial ability. Silver is known to have a considerably low toxicity to human cells and has long been used as a wide-ranged antimicrobial agent in therapeutic applications. For example, silver in various forms (silver nitrate, silver sulfadiazine, silver foams, and silvercel) have all been used as the topical antimicrobial agents for treating second- and third-degree burns [24] [25] [26] [27] [28]. Another variable we explored was nanoparticles. Nanoparticles have the advantage of possessing dimensions on the nanometer-scale as well as metallic nanoparticles having antibacterial properties against Gram-positive and Gram-negative bacteria. Studies suggest that this is likely because of their oxidative stress induction, metal ion release, or non-oxidative mechanisms [29] [30] [31].

Because of the antimicrobial property of silver and nanoparticles, it is not surprising to see that silver nanoparticles (AgNPs) exert great antimicrobial properties against several types of microorganisms including oral bacteria in many studies [32] [33] [34] [35]. For this reason, AgNPs have been reported to be effective in preventing dental caries (cavities caused by bacteria) and periodontal diseases [36] [37] [38]. In this study, we demonstrated that using simple electrodeposition procedures, AgNPs could be coated onto the titanium implants to prevent bacteria growth around the implants. In a broader perspective, electrodeposition of AgNPs possesses the potential to neutralize the complication of infection in dental implants. Notably, our results showed that cI3D implants generally yielded higher antibacterial activities than did the solid implants when coated with AgNPs. This is likely because the AgNPs are deposited into the porous structures of cI3D implants, which then can be slowly and sustainably 
released to increase the antibacterial activity. In addition, others have reported that AgNPs can be used to coat the surface of titanium implants for promoting bone tissue formation and mineralization [39] [40]. Therefore, in addition to the prevention of infection, coating implants with AgNPs may also enhance osseointegration.

\section{Conclusion Remark}

This study shows that implants with interconnecting 3D structures can better retain antimicrobial agents to prevent infection compared to solid implants. One may concern that the porous I3D structure may weaken the implant. To address the concern, the size and number of pores can be carefully considered such that the weakening would not reach the critical point. The reported median shear strength for teeth is $38.99 \mathrm{MPa}$ [41]. In comparison, Titanium alloy (Ti-6Al-4V) is an order of magnitude stronger, which has a shear strength of $760 \mathrm{MPa}$. Therefore, even with many pores, a properly designed implant can still easily meet the strength requirement. We previously reported that the porous structure of I3D implants could serve as a depository for chemoattractant to recruit stem cells for osteogenesis to enhance osseointegration. Furthermore, the I3D structures allow bone ingrowth to fill the pores, which may further enhance osseointegration and fortifying the implant [12]. Thus, the pores incorporated into the implant may not significantly weaken the I3D implants once full osseointegration is established. It is well known that osseointegration is critical for the success of bone and dental implant surgeries. One of the major causes of failure for osseointegration is peri-implantitis. With the results of this study, we found that I3D structure might also serve as a depository for antimicrobial agents to prevent bacterial infection and attachment to the implants, which are believed to be the major culprits to cause the peri-implantitis. Thus, further improving the success of implant surgeries is made possible by using I3D implants coated with chemoattractant and antimicrobial agents simultaneously to enhance osseointegration and prevent peri-implantitis. Our in vitro studies provided evidence that I3D implants could be clinically valuable. A clinical trial is needed to test the I3D implants in the future. Practically, dentists may simply dip the I3D implants into an antibiotic solution before implant installation during the surgery, or anti-microbial agent coating, e.g., AgNPs coating may be integrated into the standard procedures for manufacturing I3D implants.

\section{Acknowledgements}

The work was supported by grants from Louisiana Board of Regents (NSF EPSCoR CIMM project under award \#OIA-1541079) and the LSU Biomedical Collaborative Research Program (LBCRP). The authors' contributions are as follows: S. Yao contributed to the conception, implant design, experiment design, data collection and interpretation, and draft of the manuscript; S. Guo performed the experiments of implant coating and antimicrobial assays, collected 
data, and was involved in drafting the manuscript. A. Ramos and Z Li assisted the antimicrobial experiments and data collection. H. Yao contributed to LPBF 3D printing for implant fabrication. J. Xu contributed to the conception of the project.

\section{Conflicts of Interest}

All authors declare no conflicts of interest.

\section{References}

[1] Ahmad, N. and Saad, N. (2012) Effects of Antibiotics on Dental Implants: A Review. Journal of Clinical Medicine Research, 4, 1-6. https://doi.org/10.4021/jocmr658w

[2] Bowen Antolin, A., Pascua Garcia, M.T. and Nasimi, A. (2007) Infections in Implantology: From Prophylaxis to Treatment. Medicina Oral, Patologia Oral, Cirugia Bucal, 12, E323-E330.

[3] Surapaneni, H., Yalamanchili, P.S., Basha, M.H., Potluri, S., Elisetti, N. and Kiran Kumar, M.V. (2016) Antibiotics in Dental Implants: A Review of Literature. Journal of Pharmacy and Bioallied Sciences, 8, S28-S31.

[4] Haas, D.A., Epstein, J.B. and Eggert, F.M. (1998) Antimicrobial Resistance: Dentistry's Role. Journal of the Canadian Dental Association, 64, 496-502.

[5] Daubert, D.M., Weinstein, B.F., Bordin, S., Leroux, B.G. and Flemming, T.F. (2015) Prevalence and Predictive Factors for Peri-Implant Disease and Implant Failure: A Cross-Sectional Analysis. Journal of Periodontology, 86, 337-347. https://doi.org/10.1902/jop.2014.140438

[6] Roos-Jansaker, A.M., Lindahl, C., Renvert, H. and Renvert, S. (2006) Nine- to Fourteen-Year Follow-Up of Implant Treatment. Part II: Presence of Peri-Implant Lesions. Journal of Clinical Periodontology, 33, 290-295. https://doi.org/10.1111/j.1600-051X.2006.00906.x

[7] Heitz-Mayfield, L.J. and Salvi, G.E. (2018) Peri-Implant Mucositis. Journal of Clinical Periodontology, 45, S237-S245. https://doi.org/10.1111/jcpe.12953

[8] Pontoriero, R., Tonelli, M.P., Carnevale, G., Mombelli, A., Nyman, S.R. and Lang, N.P. (1994) Experimentally Induced Peri-Implant Mucositis. A Clinical Study in Humans. Clinical Oral Implants Research 5, 254-259. https://doi.org/10.1034/j.1600-0501.1994.050409.x

[9] Leonhardt, A., Adolfsson, B., Lekholm, U., Wikström, M. and Dahlen, G. (1993) A Longitudinal Microbiological Study on Osseointegrated Titanium Implants in Partially Edentulotis Patients. Clinical Oral Implants Research 4, 113-120. https://doi.org/10.1034/j.1600-0501.1993.040301.x

[10] Quirynen, M., Vogels, R., Peeters, W., van Steenberghe, D., Naert, I. and Haffajee, A. (2006) Dynamics of Initial Subgingival Colonization of "Pristine" Peri-Implant Pockets. Clinical Oral Implants Research, 17, 25-37. https://doi.org/10.1111/j.1600-0501.2005.01194.x

[11] Mumcu, E. and Fadhil, S.M.T. (2018) The Role of Etiologic Factors Causing Peri-Implantitis: A Current Update. Acta Scientific Dental Sciences, 2, 19-26.

[12] Bollman, M., Malbrue, R., Li, C., Yao, H., Guo, S. and Yao, S. (2020) Improvement of Osseointegration by Recruiting Stem Cells to Titanium Implants Fabricated with 3D Printing. Annals of the New York Academy of Sciences, 1463, 37-44. https://doi.org/10.1111/nyas.14251 
[13] Sirivisoot, S., Pareta, R. and Webster, T.J. (2011) Electrically Controlled Drug Release from Nanostructured Polypyrrole Coated on Titanium. Nanotechnology, 22, 085101. https://doi.org/10.1088/0957-4484/22/8/085101

[14] Karlowsky, J., Ferguson, J. and Zhanel, G. (1993) A Review of Commonly Prescribed Oral Antibiotics in General Dentistry. Journal of the Canadian Dental Association, 59, 292-294, 297-300.

[15] Dakal, T.C., Kumar, A., Majumdar, R.S. and Yadav, V. (2016) Mechanistic Basis of Antimicrobial Actions of Silver Nanoparticles. Frontiers in Microbiology, 7, 1831. https://doi.org/10.3389/fmicb.2016.01831

[16] Morones, J.R., Elechiguerra, J.L., Camacho, A., Holt, K., Kouri, J.B., Ramirez, J.T. and Yacaman, M.J. (2005) The Bactericidal Effect of Silver Nanoparticles. Nanotechnology, 16, 2346-2353. https://doi.org/10.1088/0957-4484/16/10/059

[17] Kim, J.S., Kuk, E., Yu, K.N., Kim, J.H., Park, S.J., Lee, H.J., Kim, S.H., Park, Y.K., Park, Y.H., Hwang, C.Y., Kim, Y.K., Lee, Y.S., Jeong, D.H. and Cho, M.H. (2007) Antimicrobial Effects of Silver Nanoparticles. Nanomedicine: Nanotechnology, Biology and Medicine, 3, 95-101. https://doi.org/10.1016/j.nano.2006.12.001

[18] Panacek, A., Kolar, M., Vecerova, R., Prucek, R., Soukupova, J., Krystof, V., Hamal, P., Zboril, R. and Kvitek, L. (2009) Antifungal Activity of Silver Nanoparticles against Candida spp. Biomaterials, 30, 6333-6340. https://doi.org/10.1016/j.biomaterials.2009.07.065

[19] Namasivayam, S., Ganesh, S. and Avimanyu, B. (2011) Evaluation of Anti-Bacterial Activity of Silver Nanoparticles Synthesized from Candida glabrata and Fusarium oxysporum. International Journal of Medicine Research, 1, 131-136.

[20] Panáček, A., Kvitek, L., Prucek, R., Kolář, M., Večeřová, R., Pizúrová, N., Sharma, V.K., Nevěčná, T.J. and Zbořil, R. (2006) Silver Colloid Nanoparticles: Synthesis, Characterization, and Their Antibacterial Activity. The Journal of Physical Chemistry $B, 110,16248-16253$. https://doi.org/10.1021/jp063826h

[21] Kim, J.-Y., Kim, S.-E., Kim, J.-E., Lee, J.-C. and Yoon, J.-Y. (2005) The Biocidal Activity of Nano-Sized Silver Particles Comparing with Silver Ion. Journal of Korean Society of Environmental Engineers, 27, 771-776.

[22] Chen, X. and Schluesener, H.J. (2008) Nanosilver: A Nanoproduct in Medical Application. Toxicology Letters, 176, 1-12. https://doi.org/10.1016/j.toxlet.2007.10.004

[23] Shivaram, A., Bose, S. and Bandyopadhyay, A. (2017) Understanding Long-Term Silver Release from Surface Modified Porous Titanium Implants. Acta Biomaterialia, 58, 550-560. https://doi.org/10.1016/j.actbio.2017.05.048

[24] Moyer, C. A., Brentano, L., Gravens, D. L., Margraf, H.W. and Monafo Jr., W.W. (1965) Treatment of Large Human Burns with 0.5 Per Cent Silver Nitrate Solution. The Archives of Surgery, 90, 812-867. https://doi.org/10.1001/archsurg.1965.01320120014002

[25] Klasen, H.J. (2000) A Historical Review of the Use of Silver in the Treatment of Burns. II. Renewed Interest for Silver. Burns, 26, 131-138.

https://doi.org/10.1016/S0305-4179(99)00116-3

[26] Jorgensen, B., Price, P., Andersen, K.E., Gottrup, F., Bech-Thomsen, N., Scanlon, E., Kirsner, R., Rheinen, H., Roed-Petersen, J., Romanelli, M., Jemec, G., Leaper, D.J., Neumann, M.H., Veraart, J., Coerper, S., Agerslev, R.H., Bendz, S.H., Larsen, J.R. and Sibbald, R.G. (2005) The Silver-Releasing foam Dressing, Contreet Foam, Promotes Faster Healing of Critically Colonised Venous Leg Ulcers: A Randomised, Controlled Trial. International Wound Journal, 2, 64-73.

https://doi.org/10.1111/j.1742-4801.2005.00084.x 
[27] Meaume, S., Vallet, D., Morere, M.N. and Teot, L. (2005) Evaluation of a Silver-Releasing Hydroalginate Dressing in Chronic Wounds with Signs of Local Infection. Journal of Wound Care, 14, 411-419. https://doi.org/10.12968/jowc.2005.14.9.26835

[28] Dai, T., Huang, Y.Y., Sharma, S.K., Hashmi, J.T., Kurup, D.B. and Hamblin, M.R. (2010) Topical Antimicrobials for Burn Wound Infections. Recent Patents on Anti-Infective Drug Discovery, 5, 124-151. https://doi.org/10.2174/157489110791233522

[29] Gurunathan, S., Han, J.W., Dayem, A.A., Eppakayala, V. and Kim, J.H. (2012) Oxidative Stress-Mediated Antibacterial Activity of Graphene Oxide and Reduced Graphene Oxide in Pseudomonas aeruginosa. International Journal of Nanomedicine, 7, 5901-5914. https://doi.org/10.2147/IJN.S37397

[30] Nagy, A., Harrison, A., Sabbani, S., Munson Jr., R.S., Dutta, P.K. and Waldman, W.J. (2011) Silver Nanoparticles Embedded in Zeolite Membranes: Release of Silver ions and Mechanism of Antibacterial Action. International Journal of Nanomedicine, 6, 1833-1852. https://doi.org/10.2147/IJN.S24019

[31] Leung, Y.H., Ng, A.M., Xu, X., Shen, Z., Gethings, L.A., Wong, M.T., Chan, C.M., Guo, M.Y., Ng, Y.H., Djurisic, A.B., Lee, P.K., Chan, W.K., Yu, L.H., Phillips, D.L., $\mathrm{Ma}$, A.P. and Leung, F.C. (2014) Mechanisms of Antibacterial Activity of MgO: Non-ROS Mediated Toxicity of MgO Nanoparticles towards Escherichia coli. Small, 10, 1171-1183. https://doi.org/10.1002/smll.201302434

[32] Şuhani, M.F., Băciuț, G., Băciuț, M., Şuhani, R. and Bran, S. (2018) Current Perspectives Regarding the Application and Incorporation of Silver Nanoparticles into Dental Biomaterials. Clujul Medical, 91, 274. https://doi.org/10.15386/cjmed-935

[33] Martinez-Robles, A.M., Loyola-Rodriguez, J.P., Zavala-Alonso, N.V., Martinez-Martinez, R.E., Ruiz, F., Lara-Castro, R.H., Donohue-Cornejo, A., Reyes-Lopez, S.Y. and Espinosa-Cristobal, L.F. (2016) Antimicrobial Properties of Biofunctionalized Silver Nanoparticles on Clinical Isolates of Streptococcus mutans and Its Serotypes. Nanomaterials (Basel), 6, 136. https://doi.org/10.3390/nano6070136

[34] Espinosa-Cristóbal, L.F., López-Ruiz, N., Cabada-Tarín, D., Reyes-López, S.Y., Zaragoza-Contreras, A., Constandse-Cortéz, D., Donohué-Cornejo, A., Tovar-Carrillo, K., Cuevas-González, J.C. and Kobayashi, T. (2018) Antiadherence and Antimicrobial Properties of Silver Nanoparticles against Streptococcus mutans on Brackets and Wires Used for Orthodontic Treatments. Journal of Nanomaterials, 2018, Article ID: 9248527. https://doi.org/10.1155/2018/9248527

[35] Halkai, K.R., Halkai, R., Mudda, J.A., Shivanna, V. and Rathod, V. (2018) Antibiofilm Efficacy of Biosynthesized Silver Nanoparticles against Endodontic-Periodontal Pathogens: An in Vitro Study. Journal of Conservative Dentistry, 21, 662-666. https://doi.org/10.4103/JCD.JCD $203 \quad 18$

[36] Mhaske, A.R., Shetty, P.C., Bhat, N.S., Ramachandra, C.S., Laxmikanth, S.M., Nagarahalli, K. and Tekale, P.D. (2015) Antiadherent and Antibacterial Properties of Stainless Steel and NiTi Orthodontic Wires Coated with Silver against Lactobacillus acidophilus-An in Vitro Study. Progress in Orthodontics, 16, Article No. 40. https://doi.org/10.1186/s40510-015-0110-0

[37] Zhang, N., Zhang, K., Xie, X., Dai, Z., Zhao, Z., Imazato, S., Al-Dulaijan, Y.A., Al-Qarni, F.D., Weir, M.D., Reynolds, M.A., Bai, Y., Wang, L. and Xu, H.H.K. (2018) Nanostructured Polymeric Materials with Protein-Repellent and Anti-Caries Properties for Dental Applications. Nanomaterials (Basel), 8, 393.

https://doi.org/10.3390/nano8060393 
[38] Emmanuel, R., Palanisamy, S., Chen, S.M., Chelladurai, K., Padmavathy, S., Saravanan, M., Prakash, P., Ajmal Ali, M. and Al-Hemaid, F.M. (2015) Antimicrobial Efficacy of Green Synthesized Drug Blended Silver Nanoparticles against Dental Caries and Periodontal Disease Causing Microorganisms. Materials Science \& Engineering C: Materials for Biological Applications, 56, 374-379.

https://doi.org/10.1016/j.msec.2015.06.033

[39] Qin, H., Zhu, C., An, Z., Jiang, Y., Zhao, Y., Wang, J., Liu, X., Hui, B., Zhang, X. and Wang, Y. (2014) Silver Nanoparticles Promote Osteogenic Differentiation of Human Urine-Derived Stem Cells at Noncytotoxic Concentrations. International Journal of Nanomedicine, 9, 2469-2478. https://doi.org/10.2147/IJN.S59753

[40] Zhang, R., Lee, P., Lui, V.C., Chen, Y., Liu, X., Lok, C.N., To, M., Yeung, K.W. and Wong, K.K. (2015) Silver Nanoparticles Promote Osteogenesis of Mesenchymal Stem Cells and Improve Bone Fracture Healing in Osteogenesis Mechanism Mouse Model. Nanomedicine, 11, 1949-1959. https://doi.org/10.1016/j.nano.2015.07.016

[41] Pioch, T. and Staehle, H.J. (1996) Experimental Investigation of the Shear Strengths of Teeth in the Region of the Dentinoenamel Junction. Quintessence International, 27, 711-714. 